\title{
La Percepción de la Motivación y Satisfacción de la Tutoría Recibida en Estudios de Posgrado
}

\author{
Claudia A. Hernández, Martha Jiménez, Eduardo Guadarrama y Ángel E. Rivera \\ Instituto Politécnico Nacional, Unidad Profesional Interdisciplinaria de Ingeniería y Ciencias Sociales y \\ Administrativas (UPIICSA), Sección de Estudios de Posgrado e Investigación. Av. Té 950, Iztacalco, Granjas \\ México, 08400, México, D.F.- México (e-mail: cahernandezh@ipn.mx, majimenez@ipn.mx, \\ eguadarramat@ipn.mx, aerivera@ipn.mx)
}

Recibido Jul. 24, 2015; Aceptado Sep. 8, 2015; Versión final Oct. 14, 2015, Publicado Abr. 2016

\begin{abstract}
Resumen
El objetivo del artículo es estudiar la percepción que estudiantes de posgrado tienen sobre la tutoría recibida en sus estudios de posgrado. Se consideran tres programas de maestría para identificar los factores que influyen en la motivación y satisfacción hacia el trabajo realizado por el director de tesis (tutor) y poder así identificar posibles áreas de mejora en beneficio de los posgrados. Es un estudio descriptivo y transversal con información recolectada de 60 egresados. Los resultados reflejan que los egresados están satisfechos con la tutoría que recibieron de parte de sus profesores directores de tesis, sintiendo una adecuada conexión emocional, retroalimentación, motivación y palabras de aliento de parte de los tutores. Sin embargo, se encontró que la falta de voluntad para continuar con el trabajo de tesis es un elemento que ha provocado que un porcentaje de ellos no hayan obtenido el grado. Se proponen estrategias de intervención como el seguimiento de las trayectorias académicas y la implementación de talleres de tesis.
\end{abstract}

Palabras clave: tutoría, dirección de tesis, estudios de posgrado, talleres de tesis

\section{Perception of the Motivation and Satisfaction Received from the Tutoring in Graduate Studies}

The objective of this article is to study the perception that graduate students have of the thesis supervisor (tutor) during his/her graduate studies. Three Master programs were considered in the study to identify factors that influence motivation and satisfaction towards the work made by the thesis advisor and being able to identify possible improvement areas in benefit of graduate studies. This is a descriptive and transversal study with information collected from 60 graduate students. The results show that graduate students are satisfied with the guide provided by their thesis advisors, feeling an adequate emotional connection, feedback, motivation and words of encouragement from the advisors. However, it was found that the lack of willingness by the students to continue with the thesis is an element causing that a significant percentage have not obtained the master degree. Intervention strategies such as tracing academic careers and the implementation of thesis workshop are proposed.

Keywords: tutoring, thesis direction, graduate studies, thesis workshop 


\section{INTRODUCCIÓN}

El Instituto Politécnico Nacional (IPN) es una institución de educación superior pública mexicana que en su oferta académica tiene programas en los niveles de licenciatura y de posgrados. En el nivel de posgrado cuenta con 141 programas de los cuales 84 son considerados Programas Nacionales de Calidad del Consejo Nacional de Ciencia y Tecnología (CONACyT) de los cuales sólo cinco pertenecen al área de Ciencias Sociales y Administrativas. La Unidad Profesional Interdisciplinaria de Ingeniería y Ciencias Sociales y Administrativas (UPIICSA) es una escuela que pertenece al Instituto Politécnico Nacional. La UPIICSA ofrece programas de posgrado desde hace más de treinta años. Los posgrados que se analizaron en la presente investigación son tres: 1) maestría en administración, 2) maestría en ingeniería industrial y 3) maestría en informática. Los tres programas cuentan con 29 profesores que tienen una edad promedio aproximada de 53 años y una antigüedad media de 26 años de servicio, la mayor parte de los académicos llevan más de veinte años asesorando tesis. Los alumnos que transitan por los programas, en su mayoría son profesionistas que se encuentran laborando en la industria y que buscan en la maestría oportunidades de adquisición de conocimiento que los lleven a obtener herramientas que les permiten tener un mejor desempeño en el ámbito laboral y por ende un crecimiento profesional. Lo anterior, hace indispensable que los estudiantes cuenten con una tutoría eficiente y de calidad, que les permita incrementar sus habilidades de investigación enfocadas a la obtención del grado académico. Además, la tutoría requiere que el estudiantado de posgrado tenga un seguimiento encauzado a proyectar su formación profesional y académica.

En la actualidad el modelo educativo del Instituto Politécnico Nacional pone más énfasis en el programa institucional de tutorías en el nivel bachillerato y licenciatura. Sin embargo, en los posgrados estudiados se lleva a cabo la consejería académica y la tutoría basada en el rol de formación en investigación, la segunda tiene como objetivo fundamental que el alumno concluya en primera instancia su trabajo de tesis, además de obtener conocimiento sobre bases metodológicas para el trabajo científico, sin embargo, en ocasiones por la exigencia de la investigación y los tiempos de terminación es posible que se llegue a perder la importancia de la trayectoria académica del estudiantado y con ello la posibilidad de apoyarlos y asesorarlos para que continúen con sus estudios, lo anterior, derivado a que los posgrados analizados son considerados profesionalizantes, en donde el contacto con la industria son un factor crucial en el aprendizaje de la solución de problemas, por lo tanto se requiere que la tutoría vaya encaminada a la estancia en la empresa y que el profesor y sus conocimientos tengan la capacidad de aportar en el proceso de enseñanza y aprendizaje, pero además puedan proyectar al estudiante a posibles especializaciones a través de los estudios doctorales.

Bajo en anterior contexto, el objetivo es estudiar la percepción que tienen los egresados de tres programas de maestría para identificar los factores que influyen en la satisfacción hacia el trabajo realizado por el director de tesis (tutor) para identificar posibles áreas de mejora en beneficio de los posgrados para así detectar áreas susceptibles de mejora e implementar estrategias que apoyen a los alumnos a contar con alternativas que les permitan continuar con el trabajo de investigación y así elevar sus indicadores de eficiencia terminal y de productividad académica.

Esta investigación se desprende de un primer seguimiento de egresados realizado en el año 2012, donde surgió la inquietud de construir un segundo estudio que ayudará a detectar la satisfacción hacia el trabajo de los tutores de tesis y algunos elementos de tipo personal que coadyuvarán a saber cómo intervienen éstos en el desarrollo y conclusión de los trabajos de investigación.

\section{LA TUTORÍA EN EL POSGRADO}

El análisis de las motivaciones para estudiar un posgrado se ha centrado en ciertos aspectos, encontrándose como principales: 1) la seguridad, b) afiliación y afecto, c) reconocimiento, d) logro, e) poder, f) conocimiento, g) autorrealización y h) cambio (Esquivel y Rojas, 2005). El conocer estas motivaciones es imprescindible, sin embargo las investigaciones se centran en estudios que abarcan la educación terciaria, aunque no sucede lo mismo con la educación a nivel de posgrado (Sánchez, Pintado, Talledo y Carcelén, 2009).

El tutor es una persona que debe poseer habilidades que permitan orientar a los alumnos a tomar decisiones adecuadas con respecto a su desempeño académico. Debe contar con la capacidad y disposición para escuchar los deseos futuros, metas, aspiraciones y expectativas. La tutoría es un proyecto que coadyuva a tener un enlace que supone la conexión de una persona con experiencia, no importando la edad con una persona de menor experiencia (Evans y Forbes, 2012). Los estudiosos han encontrado que el apoyo mutuo y las relaciones saludables con los directores de tesis coadyuvan a la labor de orientación de estudiantes de posgrado (Lechuga, 2011). De esta forma, la tutoría es considerada como un proceso 
sociocognoscitivo orientado a transformar a los estudiantes poco experimentados en personas calificadas para resolver problemas mediante su incorporación a redes de investigación en el campo de estudio (De la Cruz, García y Abreu, 2006). La tutoría es una relación interactiva de intercambio profesional entre individuos con diferentes niveles de experiencia y conocimiento, siendo función del más experimentado (mentor), el guiar y asistir al estudiante en formación, con el propósito de promover su desarrollo profesional. El tutor necesita tener cualidades de liderazgo, conocimiento y destrezas personales que permitan desarrollar un modelo de crecimiento para ambos actores (Díaz y Bastias, 2012).

El papel que tiene que desempeñar un tutor se resumen en tres categorías: 1) facilitar un ambiente de aprendizaje que satisfaga las necesidades de aprendizaje de cada estudiante, 2) proporcionar retroalimentación positiva y constructiva sobre el progreso del estudiante y su rendimiento, y 3) coadyuvar en el desarrollo intelectual que resultará en aprendizaje de alta calidad estudiantil (Retna, Chong y Cavana, 2009). Por tanto, el director de tesis en el posgrado debe desarrollar la función de supervisión efectiva en forma de tutoría, la que en relación con el desarrollo de la tesis de grado implica nueve funciones que son: 1) evaluación temprana y realista de las necesidades del estudiante, 2) foco pedagógico, 3) clarificación de expectativas y límites, 4) desafío académico y valoración, 5) gestión del conocimiento, 6) reuniones frecuentes, 7) revisión del trabajo escrito, 8) retroalimentación inmediata y constructiva, y 9) advertencia de las señales en peligro (Difabio, De Anglat, 2011).

La tutoría orientada a la dirección de tesis analizada desde la perspectiva de los autores De la Cruz, García y Abreu (2006), es aquella que conduce al estudiante en el proceso de búsqueda, análisis, gestión y evaluación de la información. Además, ayuda a emprender la investigación, implementar métodos adecuados por medio de herramientas y estrategias que proporcionen la búsqueda a las preguntas de la investigación. La tutoría permite que el alumno analice e interprete los resultados contrastándolos con la teoría, propiciando que identifique los alcances y limitaciones del estudio así como nuevas hipótesis, otros problemas de investigación y posibles aplicaciones.

La tutoría también denominada supervisión es un espacio privado en donde existe una relación multidimensional entre un profesor y un estudiante, el seguimiento debe de ser un proceso que facilite los pasos a seguir para que los alumnos logren el objetivo de obtener el grado académico, así como apoyarlos en insertarlos en la práctica académica, debido a que en un futuro ellos serán los formadores, de tal manera que la consejería se convierte en un proceso que es capaz de mirar a los estudiantes como seres humanos que pueden desarrollar habilidades que les ayudan a mantener un equilibrio en la dimensión cognitiva, espiritual, emocional y física (Sidhu, Kaur, Chan y Lee, 2015). La tesis de maestría es un trabajo que debe ser capaz de reflejar la integración de una serie de competencias que los estudiantes han obtenido durante su formación, por lo tanto, la capacidad de respuesta con respecto a los proceso de metodología y de dudas sobre contenido es fundamental para llevar a buen término la conclusión del trabajo (Semeijn, Semeijn y Gelderman, 2009).

La tutoría además de mejorar habilidades en los estudiantes que se encuentran realizando sus trabajos de tesis, para los estudiantes que aspiran a seguir una carrera en el mundo profesional o bien en las organizaciones estructuradas por niveles los profesores influyen en su formación como futuros mentores, (Horowitz y Christhoper, 2013; Ralph y Zami, 2011). Las necesidades y motivaciones cambian dependiendo de la etapa en la que se encuentre el alumno en sus estudios de posgrado, la persistencia es una de los elementos que el estudiante necesitará en cada fase del programa que curse, pero en la etapa final del proceso queda claro que la relación profesor y alumno es fundamental para llevar a buen término la conclusión de los estudios (Tinto, 1993).

En el posgrado las funciones de los tutores contemplan una serie de tareas complejas, siendo las más importantes la dirección del trabajo de investigación y el trabajo como docente (Cruz, Díaz Barriga y Abreu, 2010). La supervisión en posgrado es considerado como una forma de tutoría, donde los estudiantes poco a poco dominan el conocimiento adecuado de investigación disciplinaria (Manathunga y Goozée, 2007). La dinámica de la dirección de tesis implica un compromiso y responsabilidad tanto para el profesor que dirige como del estudiante, la conexión es interesante cuando ambos tienen interés en el proyecto de investigación y derivado de ellos, ambos descubren cuestiones interesantes que les permite que ambos avancen en el proceso (Fresán y Romo, 2011).

La tutoría en los posgrados se ha explorado desde diferentes enfoques, los autores Flores y Abreu (2012) realizaron una identificación sobre los principales atributos de los tutores desde la perspectiva de estudiantes de maestría y doctorado y hallaron que los alumnos definen a los tutores por sus rasgos de personalidad, profesionalismo y comportamiento ético, así como la comunicación y la confianza. Por su parte, Cruz, Díaz-Barriga y Abreu (2010) elaboran rúbricas que establecen diferentes competencias y sus 
niveles de ejecución deseables en el proceso tutorial. La enseñanza tutorial en el posgrado tiene propósitos diferentes a los que persiguen en el pregrado, el proceso educativo está orientado a la fundamentación, diseño y ejecución de una investigación, es decir, a la generación de nuevos conocimientos (Martínez, Laguna, García, Vázquez y Rodríguez, 2005). También se ha estudiado las funciones del tutor en el desarrollo de tesis, encontrándose que las actividades se centran en la evaluación temprana y realista de las necesidades del estudiante, foco pedagógico, clarificación de expectativas y límites, desafío académico y valoración, gestión del conocimiento, reuniones frecuentes y retroalimentación inmediata (Difabio, 2011). Por su lado, los autores como García, Laguna, Campos, Ruíz y Martínez (2001) lograron encontrar nueve funciones esenciales con sus respectivas actividades y competencias académicas de los tutores de posgrado. De igual manera, se ha indagado los efectos de las tutorías en los conocimientos, habilidades y el control del aprendizaje que adquieren los alumnos (Mohd, Hassan, Wan, Alima, Siew y Rosheny, 2012; Lin y Yi-ping, 2012), también la relación de los supervisores y el estudiantado en programas de posgrado (Lee Yen y Evans, 2012; Manathunga y Goozée, 2007; Wichmann, Wogensen, Wogensen, y Mulvany, 2012); la calidad y los efectos en el estudiante de la retroalimentación escrita que reciben de parte de sus tutores (Weaver, 2006), análisis de los roles, responsabilidades y relaciones de tutores, así como a los alumnos para el establecimiento de un contexto de aprendizaje centrado en el mismo (Chi, Murphy y Jenkins, 2002; Lechuga, 2011).

Los estudios señalan que la educación actual en el posgrado se confronta con las nuevas demandas sociales y por lo tanto no puede limitarse la formación de maestros y doctores a un proyecto de investigación puntual, sino que se exige el desempeño eficaz en el mundo real (Cruz, Díaz-Barriga, Abreu, 2010). Así, el mentor se conceptualiza como una persona que forma parte de una red de ayuda, que proporciona apoyo emocional y profesional (Ensher y Murphy, 2005). En un contexto contemporáneo, la tutoría es la relación entre el mentor y el aprendiz, además de ser un proceso que involucra a dos o más personas a trabajar juntas para desarrollar las habilidades de un individuo (Marti y Sifers, 2012).

\section{METODOLOGÍA}

El método es descriptivo de tipo transversal. La obtención de datos se realizó mediante la construcción de un cuestionario, el cual se compone de 23 reactivos, distribuidos en dos bloques: 1) motivación intrínseca y 2) satisfacción percibida sobre el trabajo realizado por el director de tesis. En la elaboración del cuestionario se tomaron en cuenta algunos factores de estudios realizados por Escalante (2010), Sánchez, Pintado, Talledo y Carcelén (2009) y Ruiz (2005). Los cuestionarios fueron enviados por correo electrónico y el tiempo de respuesta por parte de los alumnos fue de siete días. Se tomaron en cuenta a los egresados entre las generaciones del 2004 al 2009. La recolección de datos se hizo en el mes de enero de 2014. La información fue analizada en el programa SPSS versión 20.

El tipo de muestra que se utilizó es no probabilística, pues la elección de los elementos no depende de la probabilidad, sino de causas relacionadas con las características de la investigación o de quien hace la muestra. Aquí, el procedimiento no es mecánico ni con base en fórmulas de probabilidad, sino que depende del proceso de toma de decisiones de un investigador y desde luego las muestra seleccionadas obedecen a otros criterios de investigación (Hernández, Fernández y Baptista, 2006). La muestra productora de datos fue de 60 personas que accedieron a responder el cuestionario.

En la investigación se diseñó un cuestionario que consta de 23 ítems y se compone de dos dimensiones: 1) motivación del alumno y 2) satisfacción sobre el trabajo realizado por el asesor de tesis en el posgrado. Del instrumento, se obtuvo una confiabilidad de alfa de Cronbach de 0.879 , la cual indica una adecuada consistencia interna de las preguntas. La escala que se utilizó fue tipo Likert, en donde $5=$ totalmente de acuerdo, $4=$ de acuerdo, $3=$ ni de acuerdo ni en desacuerdo, $2=$ en desacuerdo y $1=$ totalmente en desacuerdo. Se llevó a cabo un análisis de factores que redujo en dos dimensiones las variables, la extracción se realizó por componentes principales (Ver Tabla 1).

\section{RESULTADOS}

\section{Factor 1. Motivación Intrínseca y Extrínseca}

De los 60 egresados, el $41 \%$ de los egresados son mujeres y el $59 \%$ hombres, de los cuales el $63 \%$ dijo tener el grado académico y el $36 \%$ dijo que no. El programa de posgrado con mayor participación fue la maestría en administración con $55 \%$, le sigue la maestría en ingeniería con el $30 \%$ y el $15 \%$ la maestría en informática, siendo este último el posgrado en donde se tiene que el $44 \%$ de los egresados no cuentan con el grado académico.

Con relación al factor uno que estudia las variables intrínsecas y extrínsecas se preguntó a los egresados si la falta de voluntad había sido un factor que hubiese influido para que en varias ocasiones dejarán la tesis, 
se elaboró un comparativo entre los egresados con grado y sin grado académico, se halló que el $74 \%$ de los egresados con grado dijeron estar en desacuerdo, sin embargo un $26 \%$ de los encuestados dijeron que estaban de acuerdo en que su falta de voluntad los había alejado de su trabajo de tesis. Por otro lado, el $59 \%$ de los participantes sin grado dijeron no estar de acuerdo, pero un dato importante es que el $41 \%$ de los exalumnos sin grado académico están de acuerdo con el hecho de que su posible falta de voluntad provocó que abandonaran el trabajo de investigación. Otra de las habilidades que ayuda a terminar los trabajos de investigación es el hecho de ser autodidacta, el $60 \%$ dijo estar totalmente de acuerdo con la aseveración.

Tabla 1. Análisis factorial de las variables que estudian las motivaciones y la satisfacción de la tutoría recibida en el posgrado

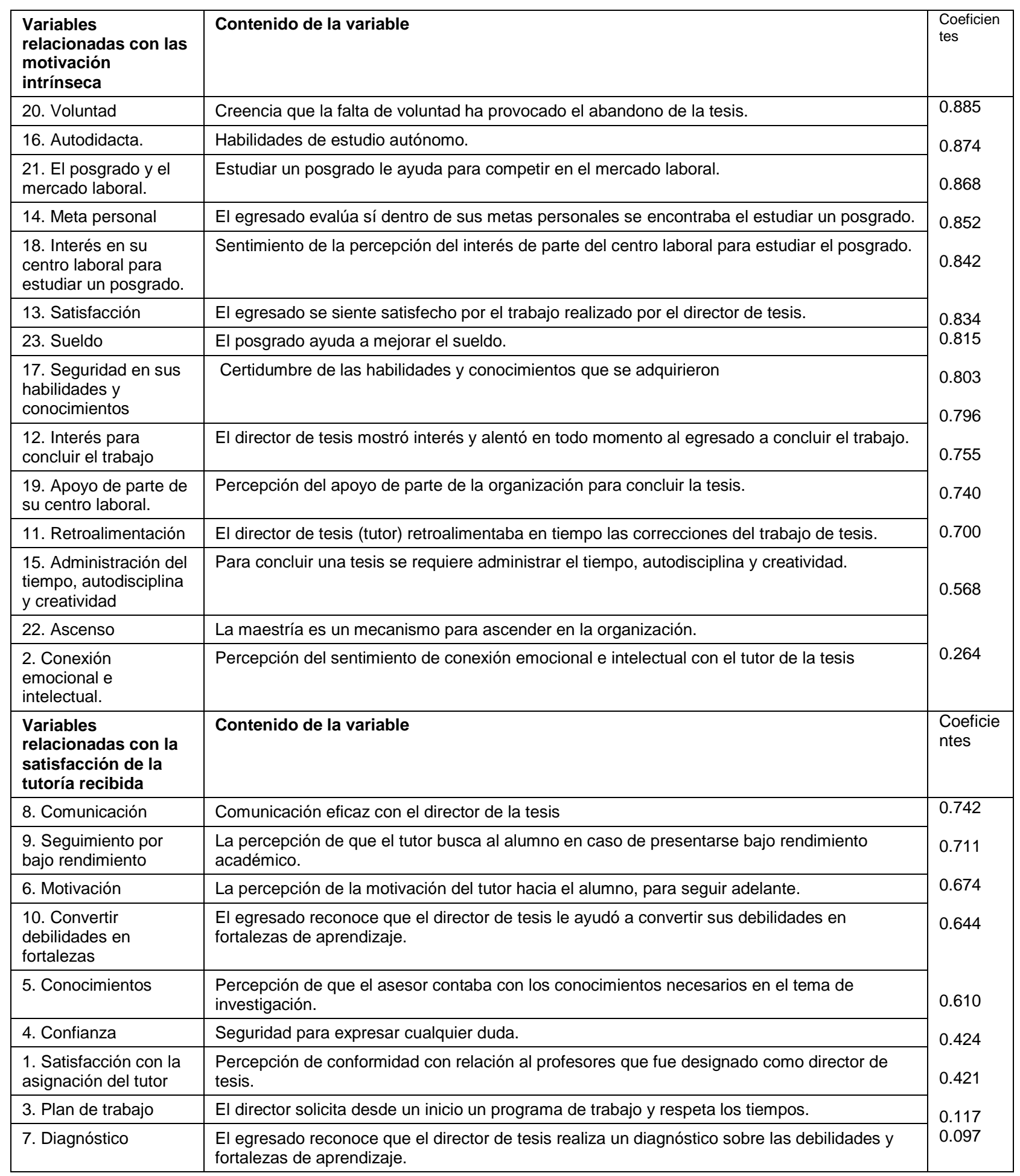


En el estudio se les cuestionó a los egresados sobre las motivaciones extrínsecas, se indagó su percepción de si el tener un posgrado ayuda a competir en el mercado laboral para los ascensos y el incremento en el sueldo, el $100 \%$ de los egresados reconocen que el contar con una maestría les ayuda a ubicarse en el mercado de trabajo, por otro lado, el $83 \%$ dice estar de acuerdo con que la maestría es un mecanismo que les ha permitido ascender en su vida profesional. También se indagó sobre el apoyo que los exalumnos habían recibido de parte de sus empleadores para estudiar el posgrado, el $74 \%$ sintió ese interés. Además, se cuestionó sobre la satisfacción percibida hacia el trabajo realizado por el director de tesis, encontrándose que el $87 \%$ se sintieron conformes. Por último, un hallazgo que se encontró fue que el $45 \%$ de los egresados señalan que el estudiar una maestría les ha ayudado a mejorar su sueldo. Asimismo, se observó que más de las tres cuartas partes de los egresados estaban totalmente de acuerdo que dentro de sus metas personales se encontraba el estudiar un posgrado. También se descubrió que el $81 \%$ de los egresados estuvieron de acuerdo en reconocer que la confianza en las habilidades y conocimientos que ellos tienen son elementos importantes que les permiten fortalecerse en todo momento durante su tránsito en el posgrado.

La motivación y el aliento que el director de tesis puede hacerle sentir al alumno, permite que él no se sienta solo en el camino que debe de recorrer, el $72 \%$ los egresados mencionan estar de acuerdo con esta aseveración. Se detectó como hallazgo que el $76 \%$ de los egresados percibieron el interés de parte de sus centros de trabajo para concluir con el trabajo de tesis, este elemento es importante ya que los egresados detectaron como oportunidad resolver una determinada problemática y la convirtieron en tema de investigación y por ende tesis de grado. La retroalimentación precisa y con tiempo del trabajo de investigación permite al alumno avanzar de forma más rápido, el $86 \%$ de los egresados consideraron que el director de tesis les entregaba las correcciones en tiempo, pero existe un $14 \%$ que no se debe de desestimar, que opina todo lo contrario. Además, en la investigación se cuestionó sobre la conclusión del trabajo de tesis y saber si ésta requiere de una adecuada administración el tiempo, autodisciplina y creatividad, el $76 \%$ dijo estar totalmente de acuerdo. La conexión emocional e intelectual es un elemento que es importante para reconocer algún tipo de empatía que el alumno sentía hacia su director de tesis. Los resultados arrojaron que el $85 \%$ sintió haber tenido ambos tipos de conexión. A su vez, el $86 \%$ estuvo de acuerdo con el hecho de que director de tesis solicitó un plan de trabajo que desde un inicio se planteaban las metas y los productos a obtener.

Se realizó un comparativo con las tres maestrías, encontrándose que los programas de maestría en administración e ingeniería obtuvieron una motivación y alta, existiendo una ligera variación con la maestría en informática en donde los egresados sintieron una motivación regular (Ver figura 1).

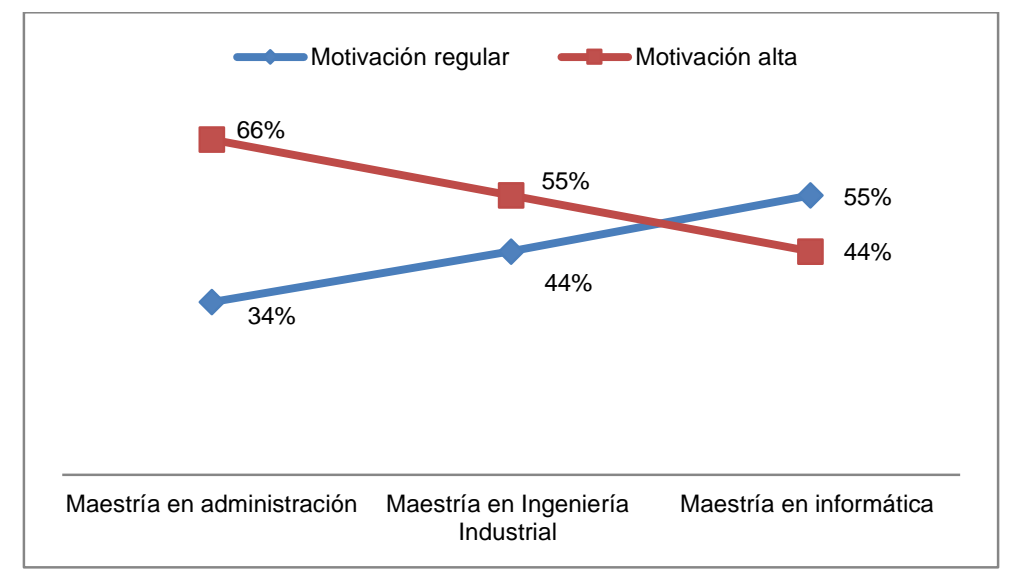

Fig. 1. Comparativo de los programas de posgrado y los niveles de motivación

\section{Factor Satisfacción con la Tutoría Recibida de Parte del Director}

El $85 \%$ percibió que la comunicación con el asesor fue la adecuada. Por otro lado, se interrogó a los ex alumnos sí su director de tesis los llegaba a buscar en caso de que detectará bajo rendimiento académico, se encontró que el $82 \%$ mencionó que sí. La función de la tutoría ayuda a reconocer debilidades en el aprendizaje y apoyar a los alumnos a fortalecerlas, se les cuestionó a los egresados sobre sí habían sentido que su asesor de tesis los había hecho reforzar áreas susceptibles de mejora con respecto al aprendizaje, el $80 \%$ menciona que sí, pero por otro lado, se encuentra un $20 \%$ que dice que no. La confianza es un factor que permite que la comunicación fluya de manera oportuna, en la investigación se encontró que el 92\% de los encuestados estaba de acuerdo con haber sentido que se daba la confianza entre el alumno y el director de la tesis. El director para que pueda coadyuvar en retroalimentar el trabajo de investigación, requiere en 
ocasiones contar con conocimientos sobre el tema que el alumno desea desarrollar, en el estudio se observó que el $73 \%$ de los egresados piensan que el tutor contaba con el saber suficiente.

Se efectúo un comparativo de los niveles de satisfacción, se observó que la maestría en informática es el posgrado en el que sus egresados señalaron estar mejor satisfechos, le sigue la maestría en administración y la ingeniería industrial.

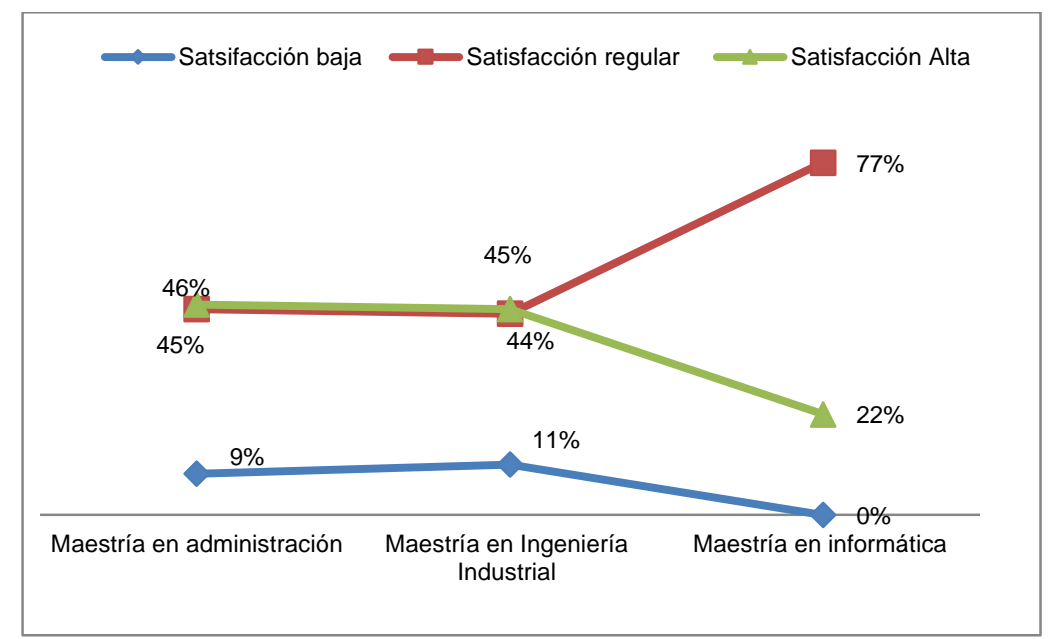

Fig. 2. Comparativo de los programas de posgrado y los niveles de satisfacción

\section{DISCUSIÓN}

El 76\% de los participantes dijeron haberse sentido conformes con la asignación de su director de tesis. Al respecto Manathunga y Goozée (2007) señalan que la supervisión en el posgrado es una tutoría con el propósito de que los alumnos poco a poco vayan dominando el conocimiento adecuado de la investigación disciplinaria; de igual forma comentan que la tutoría es una herramienta que permite a los alumnos fortalecer sus habilidades no sólo de investigación sino de trabajo autónomo. El 85\% de los egresados participantes dijeron haber sentido la conexión emocional e intelectual con su tutor, de acuerdo con De la Cruz, García y Abreu (2006) este tipo de conexión favorece al desarrollo personal y académico del profesor, el asesor debe ayudar al estudiante a manejar la incertidumbre y el estrés propios del proceso de investigación y del ejercicio profesional, además lo auxilia a manejar sus emociones derivadas de la presentación y debate de sus ideas en diferentes foros, apoyándolo especialmente cuando se confronte con situaciones críticas durante el proceso formativo como evaluaciones inherentes al desarrollo profesional o de investigación.

La confianza es un componente de la motivación, se encontró que el $92 \%$ de los participantes dijeron haber sentido este tipo de confianza con sus directores de tesis. Los autores Stefani, Tariq, Heylings y Butcher (2006) señalan que para mejorar el aprendizaje del estudiante es esencial establecer un clima de diálogo abierto entre los asesores y los estudiantes, para compartir las concepciones de aprendizaje y para asegurar que la evaluación de cualquier tarea de aprendizaje es acorde con los objetivos y resultados de aprendizaje. Por lo tanto, el propiciar ambientes agradables y plagados de confianza coadyuva a tener una interacción adecuada entre los actores involucrados en el proceso de la generación del trabajo científico.

Estos resultados son los primeros indicios que en primera instancia ayudarán a implementar una evaluación por lo menos semestral a los asesores de tesis, cabe señalar que actualmente no existe este tipo de seguimiento que permita determinar el desempeño de los directores de tesis. Además apoyará en proponer el diseño de programas de capacitación pedagógica para los docentes que en este momento están llevando a cabo la supervisión de tesis. Los programas de posgrado tienen que trabajar en fortalecer las competencias formativas-socializadoras y las competencias interpersonales de los directores de tesis. Los resultados que se detallaron en el presente estudio, orientan a pensar en estructurar un programa de apoyo entre pares en los posgrados. Es posible que el ejercicio de este tipo de tutorías permita que los alumnos de recién ingreso fortalezcan la autoestima y se sientan protegidos y orientados en el hecho de entender el proceso de elaboración de tesis. Las investigaciones realizadas por Hortsmanshof y Conrad (2003), enfatizan en fortalecer actividades relacionadas con: a) la gestión del tiempo y fijación de metas, b) la búsqueda bibliográfica, revisión y la gestión de base de datos, c) la formulación de preguntas de investigación y la selección de metodologías apropiadas y d) la estructuración del protocolo de investigación. 
Otra de las estrategias de intervención que surgieron después de la reflexión fue pensar en la implementación de laboratorios de tesis los cuales sean capaces de dar capacitación a los profesores y los alumnos, de tal forma que se logren incrementar los porcentajes de producción académica y científica colaborativa, además de desarrollar habilidades en la búsqueda de bibliografía, gestión de bases de datos, redacción de tesis y de artículos que permitirán que los alumnos y profesores trabajen en conjunto (Mcclure, 2007). También es necesario planear seminarios de investigación que tengan como propósito que los académicos y los alumnos se acerquen para desarrollar competencias en la elaboración de protocolos de investigación, capítulos de libros, ponencias y estimular su participación en eventos académicos.

Los resultados encontrados indican que los egresados están satisfechos con la tutoría recibida durante sus estudios de posgrado, sin embargo se sugiere que se trabajen alternativas de graduación distintas al trabajo riguroso que implica el desarrollo de una tesis, las opciones que se podrían trabajar puede ser la tesina y los estudios de caso y los exámenes de conocimiento, además de implementar los laboratorios de tesis que serán apoyados por cursos propedéuticos de metodologías de la investigación que permitirían que desde un inicio los estudiantes identificaran y desarrollaran un protocolo de investigación, apoyados del uso eficiente de bases de datos de búsqueda avanzada de artículos de investigación. Las implicaciones para los posgrados están encaminadas en programar una actualización de los planes de estudio, enfocados a la detección de necesidades de los egresados y de los empleadores, con ello los tutores pueden visualizar a mayor detalle las demandas actuales del mercado de trabajo. También se debe motivar a los profesorestutores a realizar estancias de investigación en la industria, con esto se pueden estructurar casos de estudio además de estimular a los estudiantes a resolver problemas reales de la industria. Asimismo, se puede implementar programas informáticos que faciliten la localización de los egresados y que apoye a la trazabilidad de su trayectoria académica, esto favorece el seguimiento de ellos.

La tutoría entre pares puede ser una herramienta que ayuda a motivar a los estudiantes durante su recorrido en los posgrados, el recibir la asesoría académica y motivacional de un par, permite que los alumnos ganen confianza y funciona como terapia en los momentos en que la falta de voluntad se desvanece y ya no se desea continuar con el trabajo de tesis. Los programas para el rescate de egresados que no tienen el grado son una estrategia que rescata a los exalumno, esto se consigue siempre y cuando exista un compromiso con todas las partes involucradas.

\section{CONCLUSIONES}

i) En general, los egresados están satisfechos con la tutoría recibida durante sus estudios de posgrado, sin embargo se deben redoblar los esfuerzos en el seguimiento de los estudiantes que son de tiempo parcial para incrementar el porcentaje de eficiencia terminal.

ii) Se deben trabajar alternativas de graduación distintas al trabajo que implica el desarrollo de una tesis, se propone el uso de la tesina, los estudios de caso y los exámenes de conocimiento.

iii) Se sugiere implementar laboratorios de tesis, apoyados por cursos de metodologías de la investigación

iv) Motivar a los profesores-tutores a realizar estancias de investigación en la industria y tomar cursos de formación pedagógica.

v) Se sugiere implementar programas informáticos que faciliten la localización de los egresados y que apoye a la trazabilidad de su trayectoria académica.

vi) Los programas para el rescate de egresados que no tienen el grado.

\section{REFERENCIAS}

Chi, K., D. Murphy y W. Jenkins (2002) The teacher's role in supporting a learner-centred learning environment: voices from a group of part-time postgraduate student in Hong Kong, International Journal of Lifelong Education, 21(5), 462-473 (2002)

Cruz, G, F., A. Díaz-Barriga y L. Abreu. La labor tutorial en los estudios de posgrado. Rúbricas para guiar su desempeño y evaluación, Perfiles Educativos, 32(130), 83-102 (2010)

De la Cruz, G., T. García y L. Abreu. Modelo integrador de la tutoría: de la dirección de tesis a la sociedad del conocimiento, Revista Mexicana de Investigación Educativa, 11(31), 1363-1388 (2006) 
Flores, C. y L.F. Abreu. Atributos de tutores de posgrado por campo disciplinario: La perspectiva de estudiantes de la Universidad Nacional Autónoma de México. Perfiles educativos, 34(138), 10-27 (2012)

Díaz, C. y C. Bastías. Una aproximación a los patrones de comunicación entre el profesor mentor y el profesor-estudiante en el contexto de la práctica pedagógica, Revista Educación XX1, 15(1), 241-263 (2012)

Difabio De Anglat, H. Las funciones del tutor de la tesis doctoral en educación. Revista Mexicana De Investigación Educativa, 16(50), 935-959 (2011)

Ensher, E. y S. Murphy. Power mentoring, How successful mentor and protégés get the most out of their relathionships, Jossey-Bass, San Francisco, California (2005)

Escalante, E. Un análisis descriptivo y fenomenológico de problemas en la elaboración de tesis de maestría, Revista Reencuentro, (57), 38-47 (2010)

Esquivel, L. y C. Rojas. Motivos de estudiantes de nuevo ingreso para estudiar un posgrado en educación, Revista Iberoamericana de Educación, 36(5) (2005)

Evans, R. y L. Forbes. Mentoring the 'net generation': faculty perspectives in health education. College Student Journal, 46(2), 397-404 (2012)

Fresán, M. y A. Romo. Programas institucionales de tutorías una propuesta de la ANUIES, Asociación Nacional de Universidades e Instituciones de Educación Superior, México (2011)

García, M., J. Laguna., J.O. Campos, R. Ruíz, y A. Martínez. Competencias académicas de los tutores del posgrado en ciencias de la tierra de la UNAM, Geos, Unión Goefísica Mexicana, 47-54 (2001)

Hernández, R., C. Fernández y P. Baptista. Metodología de la investigación, cuarta edición, McGraw Hill, México (2006)

Horowitz, J y K. Christopher. The Research Mentoring Program: Serving the Needs of Graduate and Undergraduate Researchers, Innovative Higher Education, 38(2), 105-116 (2013)

Hortsmanshof, L., y L. Conrad. Postgraduate peer support programme: Enhancing community. In Learning for an Unknown Future. Proceedings of the 26th HERDSA Annual Conference (2003)

Instituto Politécnico Nacional. Un nuevo modelo educativo para el IPN, materiales para la reforma, Instituto Politécnico Nacional, México (2003)

Lechuga, V. Faculty-graduate student mentoring relationships: mentors' perceived roles and responsibilities, Higher Education, 62(6), 757-771 (2011)

Lee Yen, M. y T. Evans. The Relationships Between Postgraduate Research Students' Psychological Attributes and Their Supervisors' Supervision Training, Procedia - Social and Behavioral Sciences, 31, 788793 (2012)

Lin, Y y A. Yi-ping. Peer mentoring among doctoral students of science and engineering in Taiwan, Asia Pacific Education Review, 13(4), 563-572 (2012)

Manathunga, C. y J. Goozée. Challenging the dual assumption of the 'always/already' autonomous student and effective supervisor, Teaching in Higher Education, 12(3), 309-322 (2007)

Martin, S. y S. Sifer. An evaluation of factors leading to mentor satisfaction with the mentoring relationship, in Children and Youth Services Review, 34(5), 940-945 (2012)

Martínez, A., J. Laguna, M.C. García, Ma. I. Vázquez y R. Rodríguez, R. Perfil de competencias del tutor de posgrado de la Universidad Autónoma de México, Universidad Autónoma de México, México (2005)

Mcclure, J. Preparing a laboratory-based thesis: Chinese international research students' experiences of supervision, Teaching in Higher Education, 10(1), 3-16 (2007) 
Mohd, N., M. W. Hassan, N. Wan, N. Alima, N. Siew y H. Rosheny. Peer-tutoring and Tertiary ESL Learners, The 8th International Language for Specific Purposes (LSP) Seminar - Aligning Theoretical Knowledge with Professional Practice. Procedia - Social and Behavioral Sciences, 66, 441-447 (2012)

Ralph, H. y J. Zami. Developing Peer Mentoring through Evaluation, Journal Innovative Higher Education, 36:41-52 (2011)

Retna, K. S., E. Chong y R.Y. Cavana. Tutors and tutorials: students' perceptions in a New Zealand university. Journal Of Higher Education Policy \& Management, 31(3), 251-260 (2009).

Ruíz, C. Enfoque estratégico de la tutoría de la tesis de grado: un modelo alternativo para aprender a investigar en el posgrado, Revista Sapiens, 6(1), 61-83 (2005)

Sánchez, J., T. Pintado, H. Talledo y S. Carcelén. La educación de posgrado en España. Un estudio empírico de la estructura de motivaciones latentes, Revista Innovar, vol. 19. 131-140 (2009)

Semeijn, J. H., J. Semeijn y K.J. Gelderman. Master Thesis Supervision. In Real Learning Opportunities at Business School and Beyond, 211-222, Springer Netherlands (2009)

Sidhu, G. K., S. Kaur, Y.F. Chan y L.F Lee. Establishing a Holistic Approach for Postgraduate Supervision. In Taylor's 7th Teaching and Learning Conference 2014 Proceedings 529-545. Springer Singapore (2015)

Stefani, L.A.J., V.N. Tariq, D.J.A. Heylings y A.C Butcher. A Comparison of Tutor and Student Conceptions of Undergraduate Research Project Work, Assessment \& Evaluation in Higher Education, 22(3), pp. 271-288 (2006)

Tinto, V. Reflexiones sobre al abandono de los estudios superiores, Perfiles educativos, vol. 18, núm. 3(7), pp. 35-52 (1993)

Weaver, M. Do students value feedback? Student perceptions of tutors' written responses, Assessment \& Evaluation in Higher Education, 31(3), 379-394 (2006).

Wichmann-Hansen, G., L. Bach, W, Eika y M. J. Mulvany. Successful PhD supervision: a two-way process. In The Researching, Teaching, and Learning Triangle, 55-64. Springer New York (2012) 